\title{
Relativistic Thomas-Fermi description of Sm isotopes at finite temperature
}

\author{
C. Providência ${ }^{1}$, D.P.Menezes ${ }^{2}$ and L. Brito ${ }^{1}$ \\ ${ }^{1}$ Centro de Física Teórica - Dep. de Física - Universidade de Coimbra \\ P-3004 - 516 Coimbra - Portugal \\ ${ }^{2}$ Dep. de Física - CFM - Universidade Federal de Santa Catarina \\ Florianópolis - SC - CP. 476 - CEP 88.040 - 900 - Brazil
}

\begin{abstract}
The caloric curve (excitation energy per particle as a function of temperature) for finite nuclei is calculated within the non-linear Walecka model for different proton fractions and different parameterizations. The results obtained are compared with published experimental data and other theoretical results. Other properties related with the droplet formation as the surface energy, neutron skin thickness and binding energy per nucleon are also displayed as a function of temperature and their behaviours are discussed. It is shown that the caloric curve is sensitive to the proton fraction and to the Coulomb interaction. At $T=0$ the droplet properties do not depend on the parameterization used. At finite temperature however, the sensitivity appears.
\end{abstract}

PACS number(s): 21.10.-k, 21.30.-x,21.65.+f,25.70.-z 


\section{Introduction}

One of the most important problems in contemporary nuclear physics and astrophysics is the determination of the properties of nuclear matter as functions of density, temperature and the neutron-proton composition. This information is important in understanding the explosion mechanism of supernova, the cooling rate of neutron stars or the processes involved in the formation of the trans-iron elements [1, 2, 3, 4, 5, 6].

Recent advances in experiments using heavy-ions at high energies and radioactive beams with large neutron or proton excess have made it possible to create not only nuclei at the limits of stability, but also a transient state of nuclear matter with appreciable isospin asymmetry, thermal excitation, and compression [7, 8, 9].

The production of several intermediate mass fragments in a short time scale during heavy ion collisions is known as nuclear multifragmentation. In multifragmentation experiments, an equilibrated system is always formed and the behaviour of the energy fluctuations suggests that the system undergoes a liquid-gas phase transition. One of the evidences of this transition is the fact that the heat-capacity exhibits a peak at a certain temperature. Although different pictures are normally used in order to describe this phase transition phenomenon [10, 11, 12], some common features are present in all of them. The caloric equation of state, which is given by the excitation energy per nucleon in terms of the thermodynamic temperature is an important quantity to be investigated in the search for a phase transition. Notice that the temperature at which phase transitions take place experimentally is not the same as the thermodynamic one.

In order to explain recent experimental results, one must study not only the ground and excited states of normal nuclei, but also nuclear states of high excitation and far from stability. In particular it is important to understand the role of the isospin degree of freedom in heavy-ion collisions at intermediate energies and the properties of nuclear matter in the region between symmetric nuclear and pure neutron matters.

The radius, thickness of neutron skins, deformation, binding energy, density distributions, and other properties of radioactive nuclei near the drip lines depend sensitively on the isospin-dependence of the nuclear equation of state (EOS). The authors of references [13] and [14] have recently stressed the possibility of extracting the EOs of asymmetric nuclear matter through the investigation of these properties as well as via reactions of neutron-rich nuclei at intermediate energies. 
The properties of neutron rich nuclei have been studied using, among others, SkyrmeHartree-Fock (SHF) and the relativistic mean-field (RMF) theories [15]. In particular it has been shown by Tanihata 113 that in SHF the saturation density essentially does not change, but in RMF it decreases rapidly as nuclear matter becomes more neutronrich. Most EOS used in the description of neutron stars and supernova are obtained from nonrelativistic models. It is therefore of interest to study the EOS, namely the inhomogeneous phase at the liquid-gas phase transition, using relativistic models. The liquid-gas phase transition is probed not only by investigating the properties of neutron stars but also through experimental results obtained in collisions involving heavy-ions and in multifragmentation experiments, already mentioned.

Nucleation process, which is known as the liquid droplet formation in a gas background, is a very old problem with many applications in different areas of physics and chemistry. Newton (1687) and Laplace (1816) dedicated themselves to understand the physics underlying the nucleation theory earlier than the formulation of the zeroth law of thermodynamics in 1913 [16]. In this work we turn into the nucleation process inside nuclear matter.

Within the framework of relativistic models, the liquid-gas phase transition in nuclear matter has been investigated at zero and finite temperatures for symmetric and asymmetric semi-infinite systems [17, 18, 19, 20, 21]. Droplet formation in the liquid-gas phase transition in cold [22, 23] and hot [24] asymmetric nuclear matter in the context of relativistic mean field theory, namely using the non-linear Walecka model (NLWM) [26] has been studied recently using a Thomas Fermi approximation. As shown in Refs. [22, 24, the optimal nuclear size of a droplet in a neutron gas is determined by a delicate balance between nuclear Coulomb and surface energies. The surface energy favors nuclei with a large number of nucleons $A$, while the nuclear Coulomb self-energy favors small nuclei.

In a previous work [25], the influence of the proton fraction in the caloric curve was calculated for nuclei obtained within the framework of the approach mentioned above. In studying multifragmentation, an input parameter called the freeze-out volume, which simulates a phase transition at constant volume, is normally used [10]. The consequences for the caloric curve of imposing thermalization in a freeze-out volume have also been discussed.

The present work extends our study, by investigating the influence of the temperature in some important quantities related to the droplet formation, such as the surface energy, 
the neutron skin thickness and the binding energy per nucleon. The importance of the Coulomb interaction and the nucleus proton fraction is investigated. The effects of using different parameterizations are also analysed. In this way, a more complete understanding of the caloric curve is obtained.

The paper is organized as follows. In section 2 the thermodynamical potential in the framework of the Thomas-Fermi approximation is calculated for the NLWM and the twophase coexistence is discussed. In section 3 we present the numerical results. Finally, in the last section the conclusions are drawn.

\section{The Thomas-Fermi Approximation in the Extended Non Linear Walecka Model}

In what follows we describe the equation of state of asymmetric matter within the framework of the relativistic non-linear Walecka model [26], [27] with the inclusion of $\rho$-mesons and the electromagnetic field. In this model the nucleons are coupled to scalar-isoscalar $\phi$, vector-isoscalar $V^{\mu}$, vector-isovector $\vec{b}^{\mu}$ meson fields and the electromagnetic field $A^{\mu}$. The lagrangian density reads:

$$
\begin{aligned}
\mathcal{L}=\bar{\psi} & {\left[\gamma_{\mu}\left(i \partial^{\mu}-g_{v} V^{\mu}-\frac{g_{\rho}}{2} \vec{\tau} \cdot \vec{b}^{\mu}-e A^{\mu} \frac{\left(1+\tau_{3}\right)}{2}\right)-\left(M-g_{s} \phi\right)\right] \psi } \\
+ & \frac{1}{2}\left(\partial_{\mu} \phi \partial^{\mu} \phi-m_{s}^{2} \phi^{2}\right)-\frac{1}{3 !} \kappa \phi^{3}-\frac{1}{4 !} \lambda \phi^{4}-\frac{1}{4} \Omega_{\mu \nu} \Omega^{\mu \nu}+\frac{1}{2} m_{v}^{2} V_{\mu} V^{\mu} \\
& +\frac{1}{4 !} \xi g_{v}^{4}\left(V_{\mu} V^{\mu}\right)^{2}-\frac{1}{4} \vec{B}_{\mu \nu} \cdot \vec{B}^{\mu \nu}+\frac{1}{2} m_{\rho}^{2} \vec{b}_{\mu} \cdot \vec{b}^{\mu}-\frac{1}{4} F_{\mu \nu} F^{\mu \nu}
\end{aligned}
$$

where $\Omega_{\mu \nu}=\partial_{\mu} V_{\nu}-\partial_{\nu} V_{\mu}, \vec{B}_{\mu \nu}=\partial_{\mu} \vec{b}_{\nu}-\partial_{\nu} \vec{b}_{\mu}-g_{\rho}\left(\vec{b}_{\mu} \times \vec{b}_{\nu}\right)$ and $F_{\mu \nu}=\partial_{\mu} A_{\nu}-\partial_{\nu} A_{\mu}$. The model comprises the following parameters: three coupling constants $g_{s}, g_{v}$ and $g_{\rho}$ of the mesons to the nucleons, the nucleon mass $M$, the masses of the mesons $m_{s}, m_{v}, m_{\rho}$, the electromagnetic coupling constant $e=\sqrt{\frac{4 \pi}{137}}$ and the self-interacting coupling constants $\kappa$, $\lambda$ and $\xi$. In this work, unless otherwise stated, we use a set of constants usually identified as NL1 [28], which is not unique, but it gives a good description of the ground state properties of many stable nuclei. The consequences of using different parameterizations is also investigated and, for this purpose, we use two other sets known as NL3 [29] and TM1 30]. The values of the constants are displayed in table 1 and the most important bulk properties obtained with these three parameterizations are displayed in table 1a. 
The basic quantity in the Thomas-Fermi approximation is the phase-space distribution function for protons and neutrons:

$$
f_{i \pm}(\mathbf{r}, \mathbf{p}, t)=\frac{1}{1+\exp \left[\left(\epsilon \mp \nu_{i}\right) / T\right]}, \quad i=p, n,
$$

where $\nu_{i}=\mu_{i}-\mathcal{V}_{i 0}$ are the effective chemical potentials with $\mu_{i}$ being the chemical potentials for particles of type $i$,

$$
\mathcal{V}_{p 0}=g_{v} V_{0}+\frac{g_{\rho}}{2} b_{0}+e A_{0}, \quad \mathcal{V}_{n 0}=g_{v} V_{0}-\frac{g_{\rho}}{2} b_{0}
$$

$\epsilon=\sqrt{p^{2}+M^{* 2}}, M^{*}=M-g_{s} \phi$ is the effective nucleon mass and $T$ is the temperature.

The classical entropy of a Fermi gas is given by

$$
S=-2 \sum_{i=p, n} \int \frac{\mathrm{d}^{3} r \mathrm{~d}^{3} p}{(2 \pi)^{3}}\left(f_{i+} \ln \left(\frac{f_{i+}}{1-f_{i+}}\right)+\ln \left(1-f_{i+}\right)+\left(f_{i+} \leftrightarrow f_{i-}\right)\right)
$$

and the thermodynamic potential is defined as

$$
\Omega=E-T S-\sum_{i=p, n} \mu_{i} N_{i}
$$

where $N_{p}, N_{n}$ are, respectively, the proton and the neutron number:

$$
N_{i}=\int \mathrm{d}^{3} r \rho_{i}(\mathbf{r}, t), \quad \rho_{i}=2 \int \frac{\mathrm{d}^{3} p}{(2 \pi)^{3}}\left(f_{i+}-f_{i-}\right), \quad i=p, n .
$$

From the above expressions we get for (4), in the static approximation,

$$
\Omega=\int \mathrm{d}^{3} r\left(\frac{1}{2}\left[(\nabla \phi)^{2}-\left(\nabla V_{0}\right)^{2}-\left(\nabla b_{0}\right)^{2}-\left(\nabla A_{0}\right)^{2}\right]-V_{e f}\right)
$$

with

$$
\begin{aligned}
& V_{e f}=-\frac{1}{2}\left[m_{s}^{2} \phi^{2}+\frac{2}{3 !} \kappa \phi^{3}+\frac{2}{4 !} \lambda \phi^{4}-m_{v}^{2} V_{0}^{2}-\frac{2}{4 !} \xi g_{v}^{4} V_{0}^{4}-m_{\rho}^{2} b_{0}^{2}\right] \\
& \quad+2 T \sum_{i} \int \frac{\mathrm{d}^{3} p}{(2 \pi)^{3}}\left[\ln \left(1+e^{-\left(\epsilon-\nu_{i}\right) / T}\right)+\ln \left(1+e^{-\left(\epsilon+\nu_{i}\right) / T}\right)\right] .
\end{aligned}
$$

The energy density reads

$$
\mathcal{E}(r, T)=2 \sum_{i} \int \frac{d^{3} p}{(2 \pi)^{3}}\left[\epsilon\left(f_{i+}+f_{i-}\right)+\mathcal{V}_{i 0}\left(f_{i+}-f_{i-}\right)\right]
$$




$$
\begin{gathered}
+\frac{1}{2}\left[(\nabla \phi)^{2}-\left(\nabla V_{0}\right)^{2}-\left(\nabla b_{0}\right)^{2}-\left(\nabla A_{0}\right)^{2}\right]+ \\
+\frac{1}{2}\left[m_{s}^{2} \phi^{2}+\frac{2}{3 !} \kappa \phi^{3}+\frac{2}{4 !} \lambda \phi^{4}-m_{v}^{2} V_{0}^{2}-\frac{2}{4 !} \xi g_{v}^{4} V_{0}^{4}-m_{\rho}^{2} b_{0}^{2}\right] .
\end{gathered}
$$

For later discussion it is convenient to separate $\mathcal{E}(r, T)$ into two parts: the nuclear contribution, $\mathcal{E}_{N}$, and the electromagnetic one; the latter is

$$
\mathcal{E}_{\text {coul. }}=2 \int \frac{d^{3} p}{(2 \pi)^{3}} \text { e } A_{0}\left(f_{p+}-f_{p-}\right)-\frac{1}{2}\left(\nabla A_{0}\right)^{2} .
$$

The fields that minimize $\Omega$ satisfy the equations

$$
\begin{gathered}
\frac{\partial V_{e f}}{\partial \phi}=-m_{s}^{2} \phi-\frac{1}{2} \kappa \phi^{2}-\frac{1}{3 !} \lambda \phi^{3}+g_{s} \rho_{s}=-\nabla^{2} \phi \\
\frac{\partial V_{e f}}{\partial V_{0}}=m_{v}^{2} V_{0}+\frac{1}{3 !} \xi g_{v}^{4} V_{0}^{3}-g_{v} \rho_{B}=\nabla^{2} V_{0} \\
\frac{\partial V_{e f}}{\partial b_{0}}=m_{\rho}^{2} b_{0}-\frac{g_{\rho}}{2} \rho_{3}=\nabla^{2} b_{0} \\
\frac{\partial V_{e f}}{\partial A_{0}}=-e \rho_{p}=\nabla^{2} A_{0}
\end{gathered}
$$

where $\rho_{B}=\rho_{p}+\rho_{n}, \rho_{3}=\rho_{p}-\rho_{n}$ and

$$
\rho_{s}=2 \sum_{i=p, n} \int \frac{\mathrm{d}^{3} p}{(2 \pi)^{3}} \frac{M^{*}}{\epsilon}\left(f_{i+}+f_{i-}\right) .
$$

These coupled differential equations are solved numerically and all relevant quantities which depend on the fields are calculated. Looking for phase transitions in binary systems such as this one requires the study of three kinds of instabilities which can occur: mechanical, diffusive and thermodynamical. The condition for mechanical stability requires

$$
\left(\frac{\partial P}{\partial \rho_{B}}\right)_{Y_{p}, T} \geq 0
$$

where $P$ is the pressure and $Y_{p}=\rho_{p} / \rho_{B}$ is the proton fraction. The condition for diffusive stability implies the inequalities

$$
\left(\frac{\partial \mu_{p}}{\partial Y_{p}}\right)_{P, T} \geq 0 \text { and }\left(\frac{\partial \mu_{n}}{\partial Y_{p}}\right)_{P, T} \leq 0 .
$$


These conditions reflect the fact that in a stable system, energy is required to increase the proton concentration at constant pressure and temperature. Thermodynamical stability is expressed by

$$
c_{v}=\left(\frac{d \varepsilon^{*}}{d T}\right)_{v, Y_{p}} \geq 0
$$

where $c_{v}$ is the specific heat and

$$
\varepsilon^{*}=\varepsilon(T)-\varepsilon(T=0),
$$

is the excitation energy per particle. The total energy per particle at temperature $T$ is given by 31

$$
\varepsilon(T)=\int \frac{\mathcal{E}(r, T)}{A} d^{3} r=\varepsilon_{N}(T)+\varepsilon_{\text {coul. }}(T),
$$

where $A$ is the total number of particles in the volume under consideration, defined at the end of section 3 , and $\varepsilon_{N}(T), \varepsilon_{\text {coul. }}(T)$ denote, respectively, the nuclear and electromagnetic contributions.

The two-phase liquid-gas coexistence is governed by the Gibbs condition

$$
\begin{aligned}
\mu_{i}\left(\rho_{p}, \rho_{n}, M^{*}\right) & =\mu_{i}\left(\rho_{p}^{\prime}, \rho_{n}^{\prime}, M^{* \prime}\right), \quad i=p, n \\
P\left(\rho_{p}, \rho_{n}, M^{*}\right) & =P\left(\rho_{p}^{\prime}, \rho_{n}^{\prime}, M^{* \prime}\right),
\end{aligned}
$$

where the primed and unprimed quantities correspond to the two different phases.

In the mean field approximation for infinite nuclear matter, the meson fields are replaced by their expectation values. From the equations of motion, they can easily be obtained [22] and the thermodynamic quantities of interest are given in terms of these meson fields. We have made use of the geometrical construction [32, 33] in order to obtain the chemical potentials in the two coexisting phases for each pressure of interest. In order to obtain the binodal section which contains points under the same pressure for different proton fractions, we have solved eqs. (18) and (19) simultaneously with the following ones:

$$
m_{s}^{2} \phi_{0}+\frac{\kappa}{2} \phi_{0}^{2}+\frac{\lambda}{6} \phi_{0}^{3}=g_{s} \rho_{s}\left(\nu_{p}, \nu_{n}, M^{*}\right)
$$

and

$$
m_{s}^{2} \phi_{0}{ }^{\prime}+\frac{\kappa}{2}{\phi_{0}}^{2}+\frac{\lambda}{6}{\phi_{0}}^{3}=g_{s} \rho_{s}\left(\nu_{p}^{\prime}, \nu_{n}^{\prime}, M^{* \prime}\right)
$$




\section{$3 \quad$ Numerical Results for Finite Systems}

The solution for the infinite system gives us the initial and boundary conditions for the program which integrates the set of coupled non-linear differential equations (9) to (12) in the Thomas-Fermi approximation. In this work the numerical calculation was carried out with the iteration procedure described in Refs. [22, 23, 24].

Some quantities of interest are outlined below. The surface energy per unit area of the droplets in the small thickness approximation is

$$
\sigma=\int_{0}^{\infty} \mathrm{d} r\left[\left(\frac{\mathrm{d} \phi}{\mathrm{d} r}\right)^{2}-\left(\frac{\mathrm{d} V_{0}}{\mathrm{~d} r}\right)^{2}-\left(\frac{\mathrm{d} b_{0}}{\mathrm{~d} r}\right)^{2}\right] .
$$

The proton and neutron radii in the spherical geometry, $R_{i}(i=p, n)$, are defined as

$$
\int_{0}^{R^{\prime}} \rho_{i}(r) r^{2} d r=\frac{1}{3}\left[\rho_{i, l} R_{i}^{3}+\rho_{i, g}\left(R^{\prime 3}-R_{i}^{3}\right)\right],
$$

where $\rho_{i, l}$ and $\rho_{i, g}$ refer to the liquid and gas density respectively and $R^{\prime}$ is the value of $r$ for which the fields and density reach their asymptotic gas values.

The neutron skin thickness is defined as [20]

$$
\Theta=R_{n}-R_{p}
$$

Other quantities of interest are the number of protons $(Z)$ and neutrons $(N)$ in the droplet, given by

$$
Z=4 \pi \int_{0}^{R^{\prime}} \rho_{p}(r) r^{2} d r-\frac{4 \pi}{3} R^{\prime 3} \rho_{p, g}
$$

and

$$
N=4 \pi \int_{0}^{R^{\prime}} \rho_{n}(r) r^{2} d r-\frac{4 \pi}{3} R^{\prime 3} \rho_{n, g} .
$$

The binding energy per nucleon is

$$
\frac{B}{A}=\varepsilon(T)-M
$$

where $A=Z+N$.

In the sequel we mainly study two nuclei: ${ }_{62}^{150} \mathrm{Sm}_{88}$ and ${ }_{62}^{166} \mathrm{Sm}_{104}$. We have chosen these two nuclei because they lie in the mass range of interest for intermediate energy heavyion collisions [0, 8, 35]. On the other hand, two isotopes with quite different number of neutrons are taken in order to study the effect of proton-neutron asymmetry. 
In figure 1, the proton density profiles are plotted with and without the electromagnetic field for the ${ }_{62}^{150} \mathrm{Sm}_{88}$ at $T=0$. The central density is smaller when the Coulomb interaction is considered because more protons are pushed off to the surface. The same behaviour is obtained for any other fixed temperature, whenever both systems, with and without the inclusion of the electromagnetic field are found. Notice, however, that the critical temperature, from which the droplet ceases to exist, is smaller when the electromagnetic field is included, as compared with systems where the Coulomb interaction is disregarded.

In figure 2, the ${ }_{62}^{150} \mathrm{Sm}_{88}$ density profiles for the protons are plotted with the inclusion of the electromagnetic field, for temperatures varying from $T=0$ to $T=6.5 \mathrm{MeV}$. The central density decreases with the increase of the temperature and consequently, the hotter the droplet, the larger it is. The same behaviour is observed when the Coulomb interaction is not included and for any other proton fraction. These results are in accordance with those obtained in [34, with a non-relativistic model.

In table 2, we show the surface energy, the proton radius, the neutron skin thickness, the central density, the binding energy per nucleon and the excitation energy per particle calculated without the inclusion of the Coulomb interaction for the ${ }_{62}^{150} \mathrm{Sm}_{88}$, which has a proton fraction equal to 0.41 . In table 3 , the same quantities are shown when calculated with the electromagnetic interaction included. In tables 4 and 5, these quantities are obtained for the ${ }_{62}^{166} \mathrm{Sm}_{104}$, which has a proton fraction of 0.37 , without and with the Coulomb interaction respectively. Examining these tables, several common trends are noticed, despite the difference in the proton fraction. In general, the surface energy, the central density and the binding energy decrease with the increase of the temperature. Inversely, the proton radius and the excitation energy per particle increase as the temperature becomes higher. Comparing tables 2 with 3 and 4 with 5 , one observes that the non inclusion of the Coulomb interaction gives rise to smaller and denser nuclei with a bigger neutron skin: the Coulomb force pushes the protons to the surface as already mentioned. It is also seen that the binding energy is always larger for the systems considered without the Coulomb interaction, in the sense that a more negative binding energy reflects a more bound state. This fact is easily explained since the Coulomb interaction tends to move protons apart and hence, diminishes the binding energy. It is however, interesting to notice that the excitation energy increases faster with temperature when the Coulomb energy is taken into account. Being a long range force one could think that there would be essentially no influence on the excitation energy. In fact, for the full calculation, one 
has $\varepsilon^{*}(T)=\varepsilon(T)-\varepsilon(0) \approx \varepsilon_{N}(T)-\varepsilon_{N}(0)$, which is explained by the long range of the Coulomb force. If the calculation is performed with no Coulomb force $(N C)$, the excitation energy $\varepsilon^{* N C}(T)=\varepsilon_{N}^{N C}(T)-\varepsilon_{N}^{N C}(0)$ contains only the nuclear contribution. In figure 3, the effect of the electromagnetic force is clear: if the Coulomb field is taken into account the excitation energy is higher, independently of the proton-neutron asymmetry, i.e. $\varepsilon^{* N C}(T)<\varepsilon^{*}(T)$. Indeed, we should not forget that the Coulomb force affects the proton distribution function and, therefore, all properties of the nucleus. As already mentioned before, we remind that in tables 3 and 5 , the last temperature $(6.5 \mathrm{MeV})$ is the one at which the droplets cease to exist within our framework, since we are not able to obtain convergence for a droplet of the size considered at higher temperatures; the same is not true in tables 2 and 4, where droplets can still be found for temperatures larger than $9 \mathrm{MeV}$.

In tables 6 and 7 , we compare for the nuclei ${ }_{62}^{150} \mathrm{Sm}_{88}$ and ${ }_{62}^{166} \mathrm{Sm}_{104}$, respectively, the results obtained for the quantities already mentioned above, when different parameterizations are used. We have considered three values of the temperature and included the Coulomb interaction. As previously noticed for the parameterization NL1, also for the other two parameterizations, the surface energy, the central density and the binding energy per particle decrease with the increase of the temperature, while the proton radius and the excitation energy per particle increase, under these conditions.

At $T=0$, the results given by all the parameterizations considered are quite similar. This reflects the fact that all the parameterizations have been fitted to the ground-state properties of stable (NL1) or stable and unstable (NL3 and TM1) nuclei. However, for finite temperatures some differences must be referred. Although at $T=0$ the surface energy is essentially the same for the 3 parameterizations, it is seen that for NL1 it reduces much faster with $T$, while in the TM1 it suffers the smallest reduction. This is true for both proton fractions, $Y_{p}=0.41$ and 0.37 . In the last case, however, $\sigma$ reduces faster with $T$ for all parameterizations.

The neutron skin thickness, $\Theta$, is not sensitive to the temperature, but it is interesting to observe that, for a given $T$, it assumes smaller values for NL3 and TM1 than for NL1. This behaviour was already discussed in [27] for $T=0$ and is related with the smaller values predicted to the symmetry energy for nuclear matter in NL3 (37.4 MeV) and in TM1 $(36.9 \mathrm{MeV})$ as compared to NL1 $(43.5 \mathrm{MeV})$. Also related with these values are the slightly higher binding energies and the slowlier increasing of the excitation energies with 
temperature for the NL3 and TM1 parameterizations.

In tables 8 and 9 several properties of isotopes with $Z=62$ are listed for $T=0$ and $T=5 \mathrm{MeV}$, respectively. One conclusion can immediately be taken: the protonneutron asymmetry makes the nuclei softer at higher temperatures and properties like the surface energy, the central density or the proton distribution radius vary much more with the number of neutrons if temperature is different from zero. It is important that a parameterization of the surface energy with temperature takes into account the isospin dependence. The decrease of the central density with the decrease of the proton fraction is typical of relativistic mean-field models. This feature is different from the prediction of the Skyrme-Hartree-Fock calculations [14], where the central density does not change with the proton fraction. We confirm that temperature has no effect on the neutron skin. In figure $₫$, we show the proton density profiles for $Z=62$ and different proton fractions, at $T=5 \mathrm{MeV}$. We observe that the proton central density increases with the proton fraction.

In figure 5 we show the caloric curves obtained with the Coulomb interaction, for ${ }_{62}^{150} \mathrm{Sm}_{88}$ (thick full line) and ${ }_{62}^{166} \mathrm{Sm}_{104}$ (thick dashed line), the excitation energies at $T=5 \mathrm{MeV}$, for different proton fractions (big triangles, from the left to the right $Y_{p}=0.33,0.35,0.37,0.39,0.41$ and 0.44 ), the experimental data from [7] (diamonds) and [35] (stars), and the Fermi-gas law $\varepsilon^{*}=1 / k T^{2}$, with $k=13.0$ (thin dashed line). We conclude that the excitation energy for ${ }^{166} \mathrm{Sm}$ (thick dashed curve), proton fraction 0.37 , increases slowlier with temperature than for ${ }^{150} \mathrm{Sm}$ (thick full curve), proton fraction 0.41. These two curves are consistent with data of 35 and a level density parameter $A / k, k=13.0$ in the Fermi gas model relation. The calculation at $T=5 \mathrm{MeV}$, for the proton fractions represented in the figure, shows that the caloric curve is sensitive to the proton-neutron ratio in the compound nucleus.

\section{Conclusions}

In summary, we have studied the surface properties and the excitation energies of arising droplets in a vapor system for temperatures up to $6.5 \mathrm{MeV}$. The droplets are described in terms of a Walecka-type model within the Thomas-Fermi approximation. It was shown that, for a fixed temperature, nuclei have a softer behaviour with the decrease of its proton fraction, namely the surface energy and the central density are smaller 
and the proton radius becomes larger. We have also concluded that the neutron skin is independent of the temperature. Another important conclusion refers to the effect of the Coulomb interaction: it reduces the central density of nuclei, as well as the neutron skin, since the protons are pushed out to the surface, and increases the excitation energy. We have also compared the behaviour with temperature of the results obtained within three different parameterizations of the non-linear Walecka model, as previously referred, NL1 [28], NL3 [29], TM1 [30]. It was shown that, at $T=0$, the three parameterizations give similar results but, at finite temperature, some of the properties investigated exhibit different behaviours: in the NL1 parameterization, the nuclei reacts in a softer way to temperature, while in the TM1 parameterization the properties of nuclei change slowlier with temperature. The excitation energies of droplets either corresponding to ${ }^{150} \mathrm{Sm}$ or ${ }^{166} \mathrm{Sm}$, for temperatures between 3 and $6.5 \mathrm{MeV}$, are consistent with the caloric curve in the Fermi gas approximation with a level density parameter $A / 13$. This result agrees with experimental data obtained in heavy-ion collisions at intermediate energies [35]. We show that the caloric curve is sensitive to the proton fraction and therefore to the symmetry term of the model used. It is also shown that close to the critical temperature the three parameterizations tested give different results. Experimentally the dependence on the proton fraction could be studied by comparing data obtained from sources with different proton fractions.

\section{Acknowledgments}

This work was partially supported by CNPq - Brazil and CFT - Portugal under the contract POCTI/35308/FIS/2000. The facilities offered by the Center for Computational Physics, University of Coimbra are warmly acknowledged.

\section{References}

[1] C.J. Pethick, D.G. Ravenhall, C.P. Lorenz, Nucl. Phys. A 584 (1995) 675.

[2] E. Baron, J. Cooperstein and S. Kahana, Phys. Rev. Lett. 55, (1985) 126; Nucl. Phys. A 440, (1985) 744.

[3] S.H. Kahana, Ann. Rev. Nucl. Part. Sci., 39 (1989) 231. 
[4] J.M. Lattimer, C.J. Pethick, M. Prakash and P. Haensel, Phys. Rev. Lett. 66 (1991) 2701.

[5] K. Sumiyoshi and H. Toki, Astro. Phys. Journal 422 (1994) 700.

[6] C. E. Rolfs and W. S. Rodney, Cauldrons in the Cosmos, The University of Chicago Press, Chicago and London, 1988.

[7] J. Phochodzalla et al., Phys. Rev. Lett. 75 (1995) 1040.

[8] J. A. Hauger et al., Phys. Rev. Lett. 77 (1996) 235.

[9] S.J. Yennello et al., in Proc. of International Workshop on Physics of Unstable Nuclear Beams, Serra Negra, Brazil, Aug. 28-31, 1996. (World Scientific, Singapore).

[10] A. Chbihi, O. Schapiro, D.H.E. Gross and S. Salou, Eur. J. Phys. A 5 (1999) 251.

[11] J.D. Frankland et al., Nucl. Phys. A 689 (2001) 940.

[12] A.B. Larionov, A.S. Botvina, M. Colonna, M. Di Toro, Nucl.Phys. A 658 (1999) 375.

[13] K. Oyamatsu, I. Tanihata, Y. Sugahara, K. Sumiyoshi and H. Toki, preprint RIKEN 199903061.

[14] B.-A. Li, C. M. Ko and W. Bauer, Int. J. Mod. Phys. E 7 (1997) 147.

[15] B.D. Serot and J.D. Walecka, Adv. Nucl. Phys. 16 (1986) 1.

[16] R.B. Lindsay, Historical Introduction in Lord Rayleigh, The Theory of Sound, Dover, New York, 1945; A. Sommerfeld, Thermodynamics and Statistical Mechanics, Academic Press, New York, 1956.

[17] H. Reinhardt and H. Schulz, Nucl. Phys. A 432 (1985) 630.

[18] H. Müller and R.M. Dreizler, Nucl. Phys. A 563 (1993) 649.

[19] D. Von Eiff, J. M. Pearson, W. Stocker and M. K.Weigel, Phys. Lett. B 324 (1994) 279.

[20] M. Centelles, M. Del Estal and X. Viñas, Nucl. Phys. A 635 (1998) 193.

[21] H. Schen, H. Toki, K. Oyamatsu and K. Sumiyoshi, Nucl. Phys. A 637 (1998) 435. 
[22] D.P. Menezes and C. Providência, Nucl. Phys. A 650 (1999) 283.

[23] G. Krein, D. P. Menezes, M. Nielsen and C. Providência, Nucl. Phys. A 674 (2000) 125.

[24] D.P. Menezes and C. Providência, Phys. Rev. C 60 (1999) 024313.

[25] D.P. Menezes and C. Providência, Caloric curve for finite nuclei in relativistic models, preprint, March 2001.

[26] J. Boguta and A. R. Bodmer, Nucl. Phys. A 292, (1977) 413; A.R. Bodmer and C.E. Price, Nucl. Phys. A 505 (1989) 123; A.R. Bodmer, Nucl. Phys. A 526 (1991) 703.

[27] Y.K. Gambir, P. Ring, A. Thimet, Ann. Phys. 198 (1990) 132; H. Berghammer, D. Vretenar and P. Ring, Phys. Lett. B 296 (1992) 290; D. Vretenar, H. Berghammer and P. Ring, Phys. Lett. B 319 (1993) 29; H. Berghammer, D. Vretenar and P. Ring, Nucl. Phys. A 560 (1993) 1014.

[28] P.-G. Reinhard, M. Rufa, J. Maruhn, W. Greiner and J. Friedrich, Z. Phys. A 323 (1986) 13.

[29] G. A. Lalazissis, J. König and P. Ring, Phys. Rev. C 55 (1997) 540.

[30] K. Sumiyoshi, H. Kuwabara, H. Toki, Nucl. Phys. A 581 (1995) 725.

[31] J. N. De, S. Gupta, S. Shlomo and S. K. Samaddar, Phys. Rev. C 55 (1997) R1641.

[32] M. Barranco and J.R. Buchler, Phys. Rev. C 22 (1980) 1729.

[33] H. Müller and B.D. Serot, Phys. Rev. C 52 (1995) 2072.

[34] S. K. Samaddar, J. N. De and S. Shlomo, Phys. Rev. Lett. 79 (1997) 4962; J. N. De, S. K. Samaddar and S. Shlomo, Nucl. Phys. A 630 (1998) 192.

[35] K. Hagel et al., Nucl. Phys. 486 (1988) 429.

[36] J. P. Bondorf, A. S. Botvina, A. S. Iljinov, I. N. Mishustin and K. Sneppen, Phys. Rep. 257 (1995) 133. 
Table 1. Sets of parameters used in this work. All masses are given in MeV.

\begin{tabular}{|c|c|c|c|c|c|c|c|c|c|c|c|}
\hline Force & {$[$ Ref. $]$} & $M$ & $m_{s}$ & $m_{v}$ & $m_{\rho}$ & $g_{s}$ & $g_{v}$ & $g_{\rho}$ & $\kappa / M$ & $\lambda$ & $\xi$ \\
\hline NL1 & {$[28]$} & 938. & 492.25 & 795.36 & 763.0 & 10.138 & 13.285 & 9.952 & 5.122 & -217.613 & 0.0 \\
\hline NL3 & {$[29]$} & 939. & 508.194 & 782.501 & 763.0 & 10.217 & 12.868 & 8.948 & 4.377 & -173.31 & 0.0 \\
\hline TM1 & {$[30]$} & 938. & 511.198 & 783.0 & 770.0 & 10.0289 & 12.6139 & 9.2644 & 3.04 & 3.7098 & 0.0169 \\
\hline
\end{tabular}


Table 1a Nuclear matter properties in the context of several parameterizations.

\begin{tabular}{|c|c|c|c|}
\hline & NL1 [28] & NL3 [29] & TM1 [30] \\
\hline$B / A(\mathrm{MeV})$ & 16.5 & 16.3 & 16.3 \\
\hline$\rho_{0}\left(\mathrm{fm}^{-3}\right)$ & 0.153 & 0.148 & 0.145 \\
\hline$K(\mathrm{MeV})$ & 211 & 272 & 281 \\
\hline$a_{\text {sym. }}(\mathrm{MeV})$ & 43.7 & 37.4 & 36.9 \\
\hline$M^{*} / M$ & 0.57 & 0.60 & 0.63 \\
\hline
\end{tabular}

Table 2. Output results given by the solution of the coupled differential equations for different temperatures without the inclusion of the electromagnetic field for ${ }_{62}^{150} \mathrm{Sm}_{88}\left(Y_{p}=\right.$ 0.41) with the NL1 parameterization.

\begin{tabular}{|c|c|c|c|c|c|c|}
\hline $\begin{array}{c}T \\
(\mathrm{MeV})\end{array}$ & $\begin{array}{c}\sigma \\
\left(\mathrm{MeV} \mathrm{fm}^{-2}\right)\end{array}$ & $\begin{array}{c}R_{p} \\
(\mathrm{fm})\end{array}$ & $\begin{array}{c}\Theta \\
(\mathrm{fm})\end{array}$ & $\begin{array}{c}\rho(0) \\
\left(\mathrm{fm}^{-3}\right)\end{array}$ & $\begin{array}{c}B / A \\
(\mathrm{MeV} / \mathrm{A})\end{array}$ & $\begin{array}{c}\varepsilon^{*}(T) \\
(\mathrm{MeV} / \mathrm{A})\end{array}$ \\
\hline \hline 0. & 1.15 & 5.88 & 0.33 & 0.161 & -11.82 & 0.00 \\
3. & 1.14 & 5.77 & 0.39 & 0.166 & -10.95 & 0.87 \\
4. & 1.06 & 5.84 & 0.37 & 0.163 & -10.64 & 1.18 \\
5. & 0.95 & 5.92 & 0.36 & 0.156 & -10.14 & 1.68 \\
6. & 0.83 & 6.04 & 0.35 & 0.147 & -9.49 & 2.33 \\
6.5 & 0.74 & 6.13 & 0.35 & 0.140 & -8.99 & 2.83 \\
7. & 0.68 & 6.22 & 0.35 & 0.137 & -8.59 & 3.23 \\
8. & 0.54 & 6.41 & 0.34 & 0.126 & -7.56 & 4.26 \\
9. & 0.40 & 6.63 & 0.36 & 0.112 & -6.33 & 5.49 \\
\hline
\end{tabular}


Table 3. Output results given by the solution of the coupled differential equations for different temperatures with the inclusion of the electromagnetic field for ${ }_{62}^{150} \mathrm{Sm}_{88}\left(Y_{p}=\right.$ 0.41) with the NL1 parameterization.

\begin{tabular}{|c|c|c|c|c|c|c|}
\hline $\begin{array}{c}T \\
(\mathrm{MeV})\end{array}$ & $\begin{array}{c}\sigma \\
\left(\mathrm{MeV} \mathrm{fm}^{-2}\right)\end{array}$ & $\begin{array}{c}R_{p} \\
(\mathrm{fm})\end{array}$ & $\begin{array}{c}\Theta \\
(\mathrm{fm})\end{array}$ & $\begin{array}{c}\rho(0) \\
\left(\mathrm{fm}^{-3}\right.\end{array}$ & $\begin{array}{c}B / A \\
(\mathrm{MeV} / \mathrm{A})\end{array}$ & $\begin{array}{c}\varepsilon^{*}(T) \\
(\mathrm{MeV} / \mathrm{A})\end{array}$ \\
\hline \hline 0. & 1.00 & 6.22 & 0.16 & 0.142 & -8.39 & 0.00 \\
3. & 0.98 & 6.23 & 0.18 & 0.140 & -7.56 & 0.83 \\
4. & 0.91 & 6.30 & 0.17 & 0.137 & -7.06 & 1.33 \\
5. & 0.81 & 6.40 & 0.16 & 0.131 & -6.37 & 2.02 \\
6. & 0.65 & 6.57 & 0.15 & 0.121 & -5.38 & 3.01 \\
6.5 & 0.57 & 6.68 & 0.14 & 0.115 & -4.78 & 3.61 \\
\hline
\end{tabular}

Table 4. Output results given by the solution of the coupled differential equations for different temperatures without the inclusion of the electromagnetic field for ${ }_{62}^{166} \operatorname{Sm}_{104}\left(Y_{p}=\right.$ 0.37) with the NL1 parameterization.

\begin{tabular}{|c|c|c|c|c|c|c|}
\hline $\begin{array}{c}T \\
(\mathrm{MeV})\end{array}$ & $\begin{array}{c}\sigma \\
\left(\mathrm{MeV} \mathrm{fm}^{-2}\right)\end{array}$ & $\begin{array}{c}R_{p} \\
(\mathrm{fm})\end{array}$ & $\begin{array}{c}\Theta \\
(\mathrm{fm})\end{array}$ & $\begin{array}{c}\rho(0) \\
\left(\mathrm{fm}^{-3}\right)\end{array}$ & $\begin{array}{c}B / A \\
(\mathrm{MeV} / \mathrm{A})\end{array}$ & $\begin{array}{c}\varepsilon^{*}(T) \\
(\mathrm{MeV} / \mathrm{A})\end{array}$ \\
\hline \hline 0. & 1.03 & 6.02 & 0.51 & 0.157 & -11.03 & 0.00 \\
2. & 0.99 & 6.03 & 0.53 & 0.154 & -10.71 & 0.32 \\
3. & 0.92 & 6.08 & 0.54 & 0.149 & -10.43 & 0.60 \\
4. & 0.84 & 6.16 & 0.54 & 0.144 & -10.12 & 0.91 \\
5. & 0.74 & 6.25 & 0.52 & 0.138 & -9.70 & 1.33 \\
6. & 0.61 & 6.39 & 0.53 & 0.129 & -9.12 & 1.91 \\
7. & 0.49 & 6.57 & 0.53 & 0.120 & -8.44 & 2.59 \\
8. & 0.35 & 6.87 & 0.55 & 0.105 & -7.57 & 3.46 \\
9. & 0.27 & 7.08 & 0.52 & 0.098 & -6.46 & 4.57 \\
\hline
\end{tabular}


Table 5. Output results given by the solution of the coupled differential equations for different temperatures with the inclusion of the electromagnetic field for ${ }_{62}^{166} \mathrm{Sm}_{104}\left(Y_{p}=\right.$ 0.37) with the NL1 parameterization.

\begin{tabular}{|c|c|c|c|c|c|c|}
\hline $\begin{array}{c}T \\
(\mathrm{MeV})\end{array}$ & $\begin{array}{c}\sigma \\
\left(\mathrm{MeV} \mathrm{fm}^{-2}\right)\end{array}$ & $\begin{array}{c}R_{p} \\
(\mathrm{fm})\end{array}$ & $\begin{array}{c}\Theta \\
(\mathrm{fm})\end{array}$ & $\begin{array}{c}\rho(0) \\
\left(\mathrm{fm}^{-3}\right)\end{array}$ & $\begin{array}{c}B / A \\
(\mathrm{MeV} / \mathrm{A})\end{array}$ & $\begin{array}{c}\varepsilon^{*}(T) \\
(\mathrm{MeV} / \mathrm{A})\end{array}$ \\
\hline \hline 0. & 0.92 & 6.40 & 0.32 & 0.138 & -7.93 & 0.00 \\
2. & 0.91 & 6.42 & 0.31 & 0.137 & -7.65 & 0.28 \\
3. & 0.86 & 6.46 & 0.32 & 0.134 & -7.26 & 0.67 \\
4. & 0.77 & 6.54 & 0.33 & 0.129 & -6.80 & 1.13 \\
5. & 0.66 & 6.67 & 0.32 & 0.122 & -6.14 & 1.79 \\
6. & 0.53 & 6.87 & 0.31 & 0.113 & -5.20 & 2.73 \\
6.5 & 0.47 & 7.00 & 0.29 & 0.108 & -4.81 & 3.12 \\
\hline
\end{tabular}


Table 6. Output results given by the solution of the coupled differential equations with the inclusion of the electromagnetic field, for ${ }_{62}^{150} \mathrm{Sm}_{88}\left(Y_{p}=0.41\right)$. For different temperatures, the results obtained with several parameterizations are shown.

\begin{tabular}{|c|c|c|c|c|c|c|c|c|c|}
\hline & \multicolumn{3}{|c|}{$\mathrm{T}=0 \mathrm{MeV}$} & \multicolumn{3}{|c|}{$\mathrm{T}=5 \mathrm{MeV}$} & \multicolumn{3}{|c|}{$\mathrm{T}=6 \mathrm{MeV}$} \\
\hline & NL1 & NL3 & TM1 & NL1 & NL3 & TM1 & NL1 & NL3 & TM1 \\
\hline$\sigma\left(\mathrm{MeV} \mathrm{fm}^{-2}\right)$ & 1.000 & 1.007 & 0.987 & 0.810 & 0.857 & 0.874 & 0.650 & 0.725 & 0.762 \\
\hline$R_{p}(\mathrm{fm})$ & 6.22 & 6.26 & 6.30 & 6.40 & 6.37 & 6.39 & 6.57 & 6.49 & 6.50 \\
\hline$\Theta(\mathrm{fm})$ & 0.16 & 0.11 & 0.09 & 0.16 & 0.12 & 0.10 & 0.15 & 0.11 & 0.10 \\
\hline$\rho(0)\left(\mathrm{fm}^{-3}\right)$ & 0.142 & 0.142 & 0.139 & 0.131 & 0.134 & 0.133 & 0.121 & 0.127 & 0.127 \\
\hline$B / A(\mathrm{MeV} / \mathrm{A})$ & -8.39 & -8.43 & -8.52 & -6.37 & -6.49 & -6.66 & -5.38 & -5.64 & -5.85 \\
\hline$\overline{\varepsilon^{\star}(T)(\mathrm{MeV} / \mathrm{A})}$ & 0.00 & 0.00 & 0.00 & 2.02 & 1.93 & 1.87 & 3.01 & 2.79 & 2.67 \\
\hline
\end{tabular}


Table 7. Output results given by the solution of the coupled differential equations with the inclusion of the electromagnetic field, for ${ }_{62}^{166} \mathrm{Sm}_{104}\left(Y_{p}=0.37\right)$. For different temperatures, the results obtained with several parameterizations are shown.

\begin{tabular}{|c|c|c|c|c|c|c|c|c|c|}
\hline & \multicolumn{3}{|c|}{$\mathrm{T}=0 \mathrm{MeV}$} & \multicolumn{3}{|c|}{$\mathrm{T}=5 \mathrm{MeV}$} & \multicolumn{3}{|c|}{$\mathrm{T}=6 \mathrm{MeV}$} \\
\hline & NL1 & NL3 & TM1 & NL1 & NL3 & TM1 & NL1 & NL3 & TM1 \\
\hline$\sigma\left(\mathrm{MeV} \mathrm{fm}^{-2}\right)$ & 0.920 & 0.933 & 0.917 & 0.660 & 0.723 & 0.742 & 0.530 & 0.615 & 0.640 \\
\hline$R_{p}(\mathrm{fm})$ & 6.40 & 6.43 & 6.49 & 6.67 & 6.62 & 6.66 & 6.87 & 6.75 & 6.76 \\
\hline$\Theta(\mathrm{fm})$ & 0.32 & 0.25 & 0.22 & 0.32 & 0.26 & 0.23 & 0.31 & 0.25 & 0.22 \\
\hline$\rho(0)\left(\mathrm{fm}^{-3}\right)$ & 0.138 & 0.139 & 0.137 & 0.122 & 0.128 & 0.127 & 0.113 & 0.120 & 0.122 \\
\hline$B / A(\mathrm{MeV} / \mathrm{A})$ & -7.93 & -8.04 & -8.11 & -6.14 & -6.30 & -6.43 & -5.20 & -5.50 & -5.67 \\
\hline$\varepsilon^{\star}(T)(\mathrm{MeV} / \mathrm{A})$ & 0.00 & 0.00 & 0.00 & 1.79 & 1.74 & 1.68 & 2.73 & 2.54 & 2.45 \\
\hline
\end{tabular}


Table 8. Output results given by the solution of the coupled differential equations for $T=0$ with the inclusion of the electromagnetic field for $Z=62$ and different number of neutrons with the NL1 parameterization.

\begin{tabular}{|c|c|c|c|c|c|c|}
\hline$N$ & $Y$ & $\begin{array}{c}\sigma \\
\left(\mathrm{MeV} \mathrm{fm}^{-2}\right)\end{array}$ & $\begin{array}{c}R_{p} \\
(\mathrm{fm})\end{array}$ & $\begin{array}{c}\Theta \\
(\mathrm{fm})\end{array}$ & $\begin{array}{c}\rho(0) \\
\left(\mathrm{fm}^{-3}\right)\end{array}$ & $\begin{array}{c}B / A \\
(\mathrm{MeV} / \mathrm{A})\end{array}$ \\
\hline \hline 78 & 0.445 & 1.05 & 6.11 & 0.05 & 0.144 & -8.59 \\
88 & 0.413 & 1.00 & 6.22 & 0.16 & 0.142 & -8.39 \\
94 & 0.397 & 0.97 & 6.29 & 0.22 & 0.141 & -8.25 \\
104 & 0.374 & 0.92 & 6.40 & 0.32 & 0.138 & -7.93 \\
112 & 0.358 & 0.87 & 6.50 & 0.39 & 0.136 & -7.76 \\
124 & 0.334 & 0.80 & 6.62 & 0.50 & 0.133 & -7.37 \\
\hline
\end{tabular}

Table 9. Output results given by the solution of the coupled differential equations for $T=5$ with the inclusion of the electromagnetic field for $Z=62$ and different number of neutrons with the NL1 parameterization.

\begin{tabular}{|c|c|c|c|c|c|c|c|}
\hline$N$ & $Y$ & $\begin{array}{c}\sigma \\
\left(\mathrm{MeV} \mathrm{fm}^{-2}\right)\end{array}$ & $\begin{array}{c}R_{p} \\
(\mathrm{fm})\end{array}$ & $\begin{array}{c}\Theta \\
(\mathrm{fm})\end{array}$ & $\begin{array}{c}\rho(0) \\
\left(\mathrm{fm}^{-3}\right)\end{array}$ & $\begin{array}{c}B / A \\
(\mathrm{MeV} / \mathrm{A})\end{array}$ & $\begin{array}{c}\varepsilon^{*}(T) \\
(\mathrm{MeV} / \mathrm{A})\end{array}$ \\
\hline \hline 78 & 0.445 & 0.87 & 6.26 & 0.04 & 0.134 & -6.32 & 2.27 \\
88 & 0.413 & 0.81 & 6.40 & 0.16 & 0.131 & -6.37 & 2.02 \\
94 & 0.396 & 0.75 & 6.46 & 0.23 & 0.128 & -6.32 & 1.93 \\
104 & 0.374 & 0.66 & 6.67 & 0.32 & 0.122 & -6.14 & 1.79 \\
112 & 0.355 & 0.55 & 6.89 & 0.39 & 0.115 & -6.13 & 1.63 \\
124 & 0.334 & 0.41 & 7.27 & 0.48 & 0.102 & -6.01 & 1.37 \\
\hline
\end{tabular}




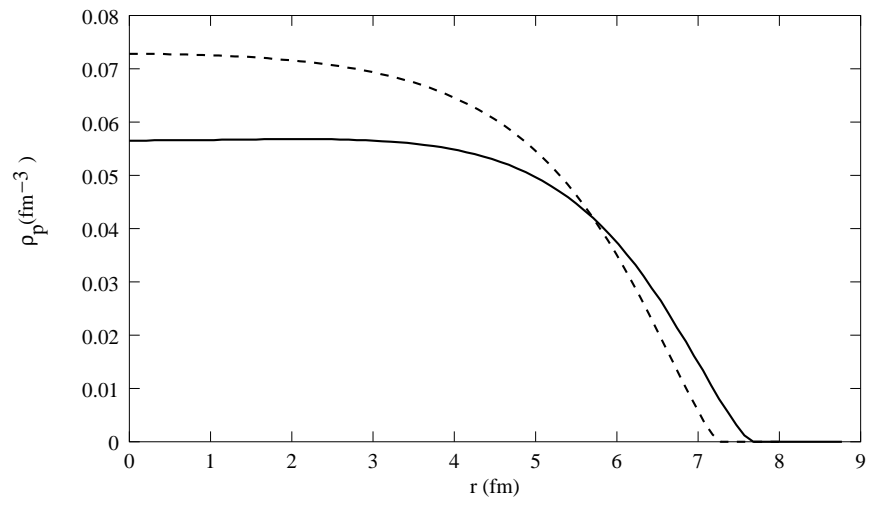

Figure 1: The ${ }_{62}^{150} \mathrm{Sm}_{88}\left(Y_{p}=0.41\right)$ density profiles for protons $\rho_{p}(r)$ in $\mathrm{fm}^{-3}$ are plotted with (solid) and without (dashed) the inclusion of the electromagnetic field for $T=0$ $\mathrm{MeV}$.

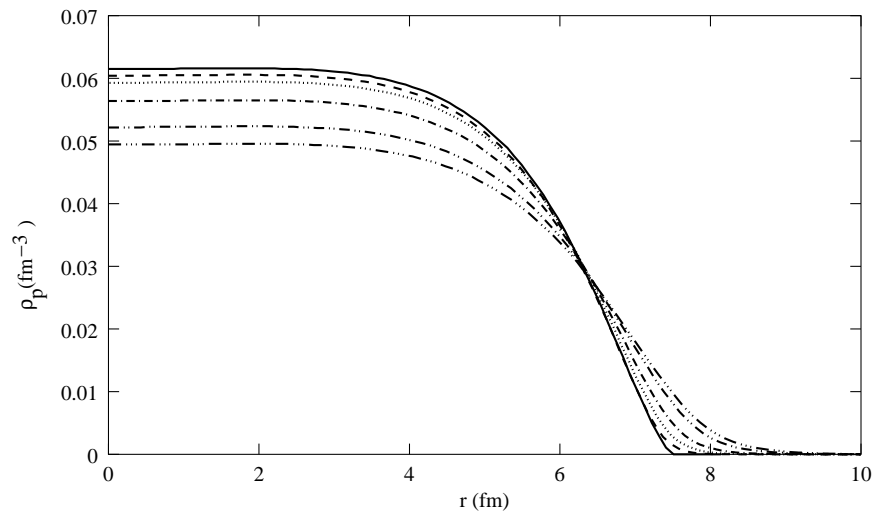

Figure 2: The ${ }_{62}^{150} \mathrm{Sm}_{88}\left(Y_{p}=0.41\right)$ density profiles for the protons $\rho_{p}(r) \mathrm{in} \mathrm{fm}^{-3}$ are plotted with the inclusion of the electromagnetic field for temperatures varying from $T=0$ (at the top, on the left) to $T=6.5 \mathrm{MeV}$ (at the bottom, on the left). 


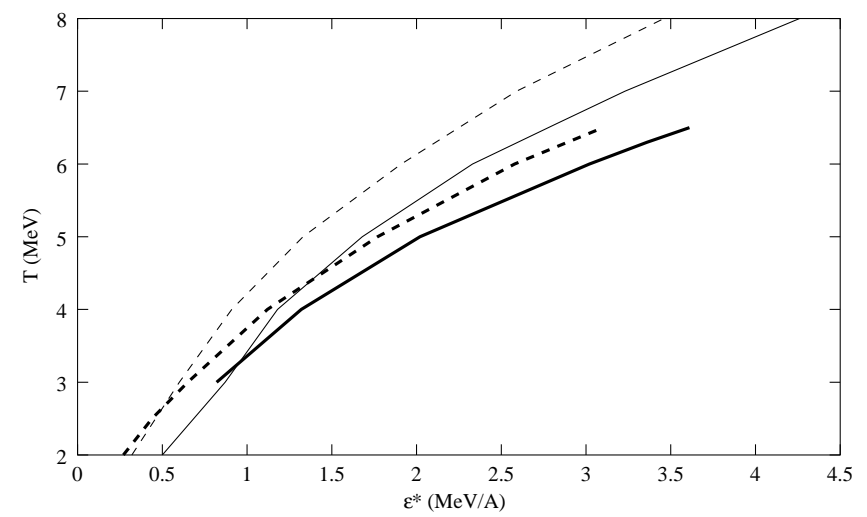

Figure 3: The caloric curves are shown for ${ }_{62}^{150} \mathrm{Sm}_{88}\left(Y_{p}=0.41\right)$ with (thick solid line) and without (thin solid line) the Coulomb interaction and for ${ }_{62}^{166} \mathrm{Sm}_{104}\left(Y_{p}=0.37\right)$ with (thick dashed line) and without (thin dashed line) the Coulomb interaction.

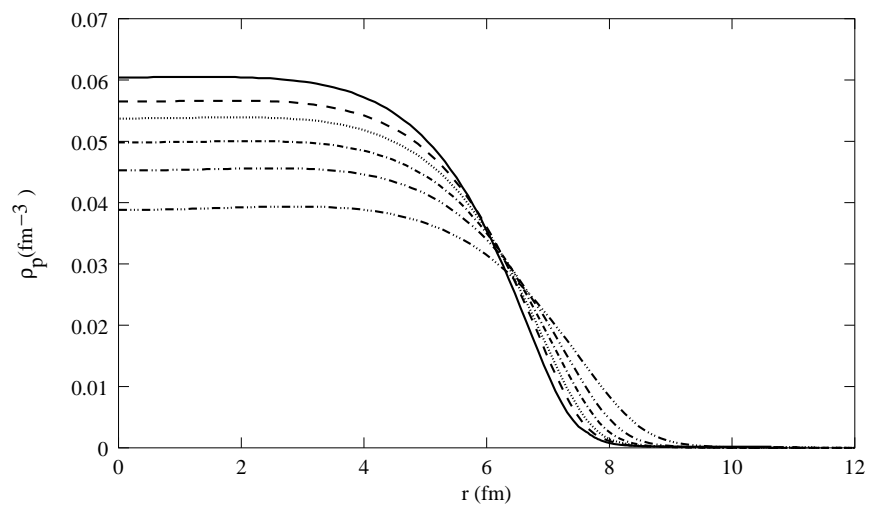

Figure 4: Density profiles for the protons $\rho_{p}(r)$ in $\mathrm{fm}^{-3}$ are plotted for $T=5 \mathrm{MeV}$ and $Y_{p}=0.44$ (solid line), $Y_{p}=0.41$ (dashed line), $Y_{p}=0.39$ (dotted line), $Y_{p}=0.37$ (dot-dashed line), $Y_{p}=0.35$ (double dot-dashed line) and $Y_{p}=0.33$ (three dot-dashed line). 


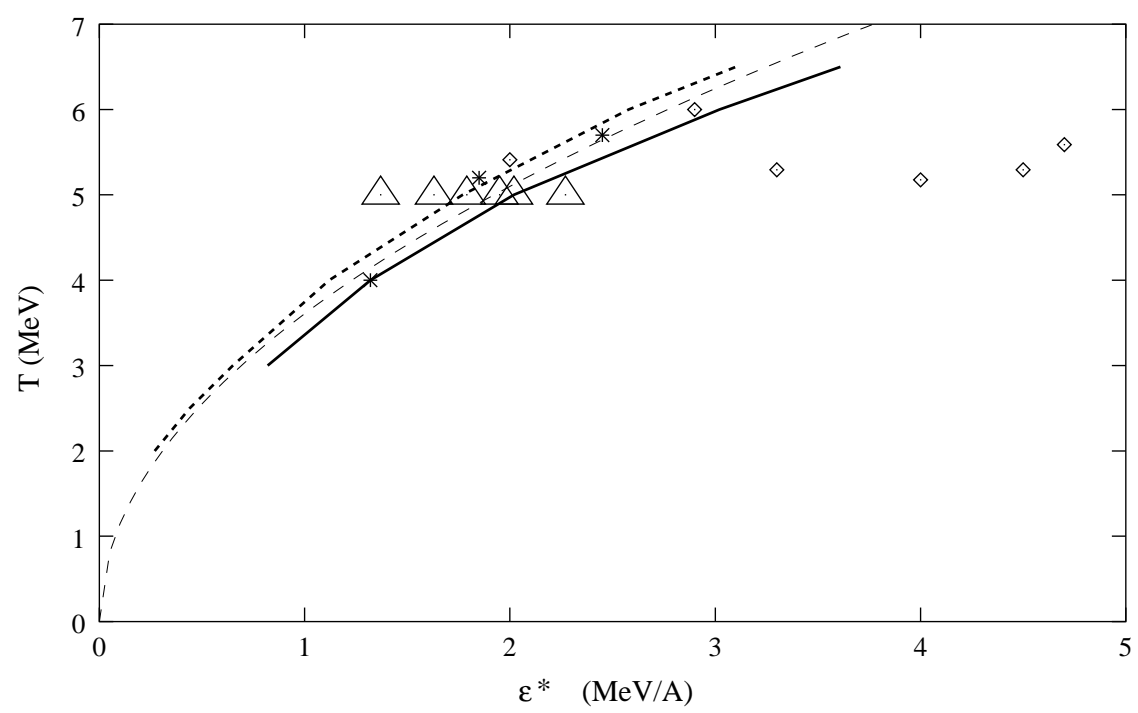

Figure 5: The caloric curves are shown for ${ }_{62}^{166} \mathrm{Sm}_{104}\left(Y_{p}=0.37\right.$ - thick dashed line) and ${ }_{62}^{150} \mathrm{Sm}_{88}\left(Y_{p}=0.41\right.$ - thick full line); the excitation energies at $T=5 \mathrm{MeV}$, for different proton fractions (big triangles, from the left to the right $Y_{p}=0.33,0.35,0.37,0.39,0.41$ and 0.44), the experimental data from [7] (diamonds) and [35] (stars), and the Fermi-gas law [35] ( $k=13.0$ - thin dashed line) are also displayed. 